\title{
Travaux pratiques sur la gestion énergétique optimale d'un bâtiment EnR
}

\author{
Benjamin Paris, Julien Eynard, Frédérik Thiery, Adama Traoré, Monique Polit, Thierry Talbert \\ benjamin.paris@univ-perp.fr, julien.eynard@univ-perp.fr, thiery@univ-perp.fr, traore@univ-perp.fr, \\ polit@univ-perp.fr, talbert@univ-perp.fr
}

Laboratoire ELIAUS, Université de Perpignan Via Domitia, 52 avenue Paul Alduy, 66860 Perpignan Cedex http://eliaus.univ-perp.fr

\section{RÉSUMÉ}

Les énergies renouvelables (EnR) prennent jour après jour une place de plus en plus importante dans notre société. Un des changements marquants de ces dernières années est l'introduction progressive des EnR (petit éolien, solaire thermique et photovoltaïque essentiellement) au sein des habitations individuelles. Cette utilisation nécessite de nouveaux systèmes de gestion électronique afin de prendre en compte la fluctuation dans le temps des différentes sources énergétiques. L'objectif de cet article est de présenter des travaux pratiques portant sur la gestion optimale de l'énergie dans un bâtiment. Pour cela les étudiants disposeront d'une maquette d'un bâtiment à l'échelle 1/27 dont ils devront contrôler la température de manière optimale. Ces TP seront intégrés au master Électronique, Automatique, Informatique et à la licence Sciences Physiques et Technologies de l'Ingénieur/parcours EEA de l'Université de Perpignan Via Domitia pour la rentrée 2008 .

Mots clés : Travaux Pratiques, Automatique, Électronique embarquée, Contrôle neuro-flou, Commande, Instrumentation, Énergies renouvelables, Bâtiment.

\section{INTRODUCTION}

L'activité anthropique du dernier siècle a profondément modifié l'écosystème dans lequel nous vivons : le changement climatique en est une des conséquences les plus visibles. Ce dernier s'explique en particulier par les émissions mondiales de gaz à effet de serre (GES), résultants majoritairement de la consommation d'énergies fossiles.

Plus précisément, ces consommations sont imputables aux différents secteurs d'activité dont le bâtiment fait partie. Il représente à lui seul $50 \%$ de l'énergie globale consommée en France ( $40 \%$ au niveau européen), c'està-dire $25 \%$ des émissions de gaz à effet de serre. Le secteur du bâtiment est le seul à voir ses consommations d'énergies finales augmenter ces dernières années, alors que tous les autres connaissent une relative diminution [1]. Cela signifie que d'importants progrès et des efforts doivent être envisagés au niveau de la performance des matériaux, de la conception, du développement des énergies renouvelables et de la gestion énergétique.

Les pouvoirs publics ne s'y sont pas trompés par le biais de la Réglementation Thermique (RT) 2005 [2] des bâtiments et des diagnostics de performances énergétiques. Les contraintes en terme de consommations conventionnelles d'énergie sont revues à la baisse, et les énergies renouvelables favorisées. A l'heure actuelle, la gestion de l'énergie dans les bâtiments reste un enjeu majeur pour l'avenir.

La recherche actuelle s'oriente vers une meilleure connaissance du fonctionnement d'un bâtiment et de l'ensemble des systèmes énergétiques installés. C'est pourquoi nous avons besoin de les instrumenter, à l'aide de capteurs intelligents spécifiques, afin d'obtenir des indicateurs de performances énergétiques. De plus, la connaissance des paramètres environnementaux est nécessaire pour définir le contexte dans lequel le bâti évolue.

\subsection{Définition de l'indicateur de performance éner- gétique}

En France, la première réglementation concernant la thermique des bâtiments est apparue après le premier choc pétrolier en 1975. Actuellement, la RT 2005 en vigueur est basée sur des normes mondiales (ISO) ou européennes (EN). Par exemple la EN 12831 présente des méthodes d'évaluation des déperditions de l'enveloppe. Tous ces documents codifient les différentes méthodes de calcul des performances (et des paramètres associés) des constructions neuves. C'est pourquoi ils sont très utiles pour connaître les indicateurs nécessaires afin d'améliorer les connaissances sur le fonctionnement d'un bâtiment d'un point de vue thermique.

En outre, la RT 2005 [3] détermine les performances énergétiques à atteindre. Il s'agit des consommations conventionnelles d'énergie représentées par les indices Th-CE 2005, pour l'ensemble d'un bâtiment et de ses équipements, en fonction de certains critères comme la localisation géographique par exemple.

Ces consommations énergétiques conventionnelles s'expriment en $\mathrm{kWh} / \mathrm{m}^{2} / \mathrm{an}$, qui représente l'indicateur global.

Cet indicateur est également utilisé dans l'existant pour le Diagnostic de Performance Energétique (DPE) [4] : calculs prenant en compte des caractéristiques des matériaux (coefficient de déperditions des vitrages, coefficient de conductivité thermique des isolants...), de la conception du bâti (indices de forme, nombre de niveaux, surface et type de murs...) en vue de définir une classe de consommation énergétique pour les bâtiments à l'image de ce qui existe dans l'électroménager (fig 1). 


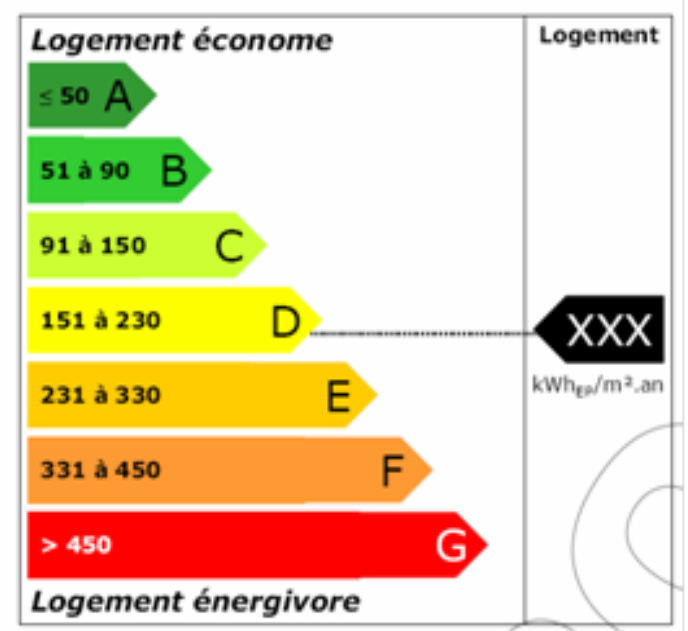

fig 1: Étiquette de performance énergétique (source ADEME)

\subsection{Les différentes sources énergétiques}

A l'heure actuelle, la quasi-totalité des bâtiments sont multi-énergies dont la plus utilisée est l'électricité. Le chauffage est le principal poste au sein d'un habitat, et reste très important dans le tertiaire. Il représente à lui seul près de $80 \%$ des émissions de GES en Europe, et consomme autant d'énergie que l'industrie ou le transport. Il existe plusieurs sources d'énergies disponibles pour le chauffage : le fioul, le gaz naturel et le propane, le bois en sont les principales.

C'est un constat qui apparaît dans le cadre législatif puisque les calculs de la RT 2005 prennent en compte le chauffage, le refroidissement, l'eau chaude sanitaire ainsi que l'éclairage. Ils caractérisent les différences de consommations d'énergie selon les postes.

\subsection{Cadre de l'étude en travaux pratiques}

Dans le cadre de la sensibilisation des étudiants aux problématiques de la gestion énergétique des bâtiments, des économies d'énergies, de l'intégration des énergies renouvelables et du contrôle optimal de systèmes complexes, nous devons disposer d'un système entièrement instrumenté, commandable, reconfigurable aisément et possédant si possible un mix énergétique. Il est difficile de disposer d'un tel bâtiment sur le campus de l'UPVD.

- Un bâtiment instrumenté complètement? Possible mais nécessite un investissement matériel et humain important.

- Un bâtiment disposant de plusieurs sources énergétiques? C'est déjà le cas de nombreux bâtiments (électrique pour le chauffage et le refroidissement, fioul pour le chauffage central) cependant aucun ne possède actuellement de sources EnR.

- Un bâtiment permettant une gestion de son énergie dans le cadre de travaux pratiques? Il est actuellement difficile de mettre à disposition des étudiants un bâtiment complet pour tester des algorithmes de régulation, sans envisager une gène de ses occupants, et de pouvoir faire un by-pass des régulations déjà intégrées dans les climatiseurs et les pompes à chaleur par exemple.

Nous avons donc réalisé une maquette d'un bâtiment, à l'échelle 1/27, ainsi qu'une électronique de mesure et de contrôle adaptée. Ces éléments sont décrits dans les paragraphes 2 et 3 . Le paragraphe 4 présente le travail que devront effectuer les étudiants en travaux pratiques. Nous terminons par les futures évolutions qui intégreront la maquette .

\section{DU BÂTIMENT RÉEL AU PROTOTYPE DE TP}

Le modèle choisi comme prototype à gérer est une habitation de $128 \mathrm{~m}^{2}$, de plein pied, avec une baie vitrée. Ces caractéristiques représentent la valeur moyenne du parc existant. Elles possèdent aussi l'avantage de la simplicité de modélisation thermique permettant de calculer les déperditions énergétiques. Grâce à sa taille réduite, la maquette permet de modifier aisément l'instrumentation par exemple l'emplacement des capteurs. Ceci amène une grande flexibilité pour définir la meilleure électronique à utiliser, l'optimisation de la position des capteurs et pour réadapter les règles d'automatique implantées. La manipulation des étudiants en est ainsi facilitée.

La conception du modèle réduit se déroule en 3 étapes :

- la mise à l'échelle physique,

- le choix des matériaux,

- le calcul des ressources/déperditions énergétiques.

\subsection{Mise à l'échelle physique}

La base de l'échelle est le rapport entre la taille réelle d'un dallage béton $(20 \mathrm{~cm}$ environ) et la taille du matériau pouvant le remplacer pour la construction de la maquette. Notre choix c'est porté sur un carrelage de $6 \mathrm{~mm}$ présentant une homogénéité semblable à celle du béton. A partir de cette échelle, on en déduit linéairement les épaisseurs des murs, des fenêtres, et de l'isolation.

\subsection{Choix des matériaux}

Le choix des matériaux répond à certaines obligations :

- l'épaisseur doit correspondre à l'échelle définie,

- le comportement thermique et hygrométrique doit être semblable à une paroi réelle.

C'est pourquoi nous avons choisi le BA13 pour les murs, le polystyrène $(4 \mathrm{~mm})$ pour l'isolation et le polyane $(4 \mu \mathrm{m})$ pour les fenêtres. Une isolation sous dallage a été réalisée, ainsi qu'une isolation intérieure des parois. Des gaines pour le passage des câble des capteurs et des actionneurs sont disposées le long des parois et dans le sol. Un pan du toit est amovible pour pouvoir modifier facilement l'instrumentation. 


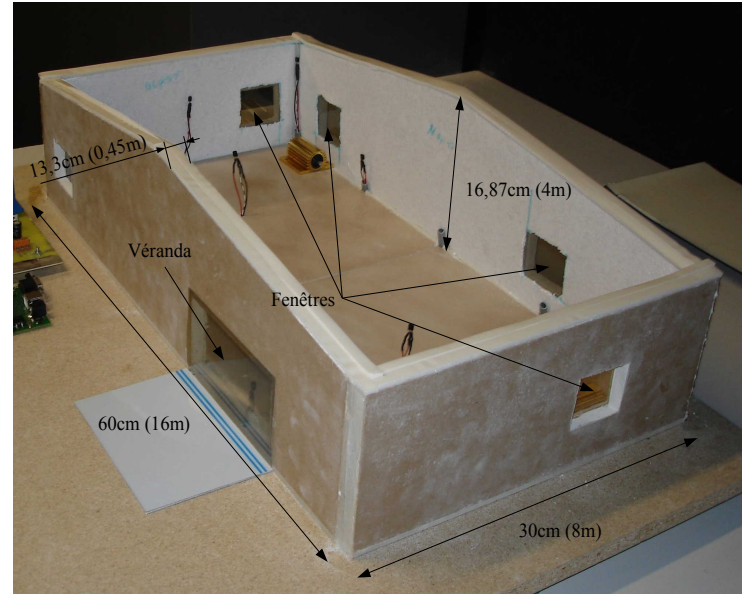

fig 2: Le prototype du bâtiment. Les dimensions grandeur nature sont données entre parenthèses.

\subsection{Calcul des ressources}

Le calcul de la puissance de chauffe nécessaire pour répondre aux besoins de chauffage se déroule de la manière suivante : les conductivités thermiques des différents matériaux $\left(\mathrm{W} / \mathrm{m}^{\circ} \mathrm{C}\right)$ sont recherchées, et associées aux épaisseurs respectives, leurs résistivités thermiques $\left(\mathrm{m}^{2 \circ} \mathrm{C} / \mathrm{W}\right)$ sont calculées. Puis, le coefficient de déperdition $\mathrm{D}_{\text {bât }}$ des parois $\left(\mathrm{W} / \mathrm{m}^{2 \circ} \mathrm{C}\right)$ est evalué pour les murs verticaux et horizontaux, ainsi que pour les parois vitrées et opaques. A partir des surfaces respectives de ces parois, on obtient les déperditions totales du bâtiment ramenées à la surface chauffée, auxquelles on ajoute une estimation des ponts thermiques. La puissance maximale de chauffe est évalué par la méthode de calcul empirique suivante :

$$
P(W)=D_{b a \hat{t}} . S .\left(T_{C}-T_{\text {ext }}\right)
$$

- $\mathrm{D}_{\text {bât }}$ : coefficient de déperdition du bâtiment W/ $\mathrm{m}^{2} \cdot{ }^{\circ} \mathrm{C}$,

- $\quad \mathrm{S}:$ surface de chauffe exprimée en $\mathrm{m}^{2}$,

- $\mathrm{T}_{\mathrm{C}}$ : température de consigne,

- $\mathrm{T}_{\mathrm{ext}}$ : température extérieure de base.

Application à la maquette : pour répondre à une température de consigne de $19^{\circ} \mathrm{C}$ et à une température extérieure de base de $-5^{\circ} \mathrm{C}$, la puissance de chauffe est estimée à $55 \mathrm{~W}$.

\section{LE SYSTÈME ÉLECTRONIQUE DE MESURE ET DE COMMANDE}

\subsection{Le système}

Le projet devait à l'origine posséder des sources EnR (solaire thermique et/ou éolien). Cependant face à la difficulté à trouver des sources réalisant ce que nous voulions (vitesse de réaction pour un TP de $3 \mathrm{~h}$, puissance adaptée à la maquette), nous avons décidé de remplacer l'ensemble des sources par des systèmes de chauffage électrique. Ceux-ci seront commandés de manière adéquate pour simuler le comportement d'une source EnR aléatoire. Le système comporte 4 cartes électroniques (fig 3) :

- la carte de commande,
- la carte des conditionneurs de signaux pour l'acquisition des données capteurs (température, humidité etc.),

- la carte d'interface entre les parties commande et puissance,

- la carte de puissance reliée aux différents actionneurs (chauffage ventilateur etc.).

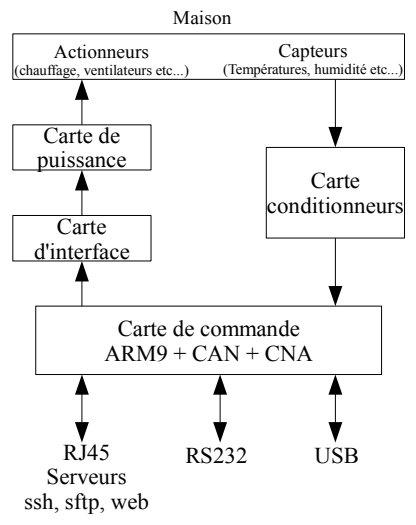

fig 3: Synoptique de l'électro-
nique

\subsection{La carte de commande}

La carte utilisée est la carte du projet GNU Armadeus $[5,6]$. Cette carte est basée sur un processeur ARM9 de la société Freescale, et possède les caractéristiques suivantes :

- 8 Mo de mémoire FLASH (type NOR),

- 16 Mo de SDRAM,

- 1 contrôleur Ethernet 10/100 Mbits Davicom (DM9000),

- 1 CAN 10 bits Max1027 (8 entrées FIFO, 300ksps, simples ou différentielles, connectées sur bus SPI $10 \mathrm{Mhz}$, consommation d'environ $700 \mathrm{~mW}$ ),

- 1 CNA 10 bits Max5821 (2 sorties, sur bus I2C compatible bus $400 \mathrm{kHz}$, consommation d'environ $375 \mathrm{~mW}$ ),

- l'ensemble du système est géré par un OS Linux.

\subsection{Les cartes d'interface et de puissance}

La maquette doit contrôler 6 actionneurs :

- 4 systèmes de chauffage de puissance différente (2 sont réservés pour simuler une source EnR et les 2 autres pour un chauffage électrique classique). Les convertisseurs assurant la chauffe sont des hacheurs 1 quadrant simple sur charge résistive. Ce sont donc 4 convertisseurs DC/DC entièrement indépendants qui sont utilisés pour la simulation des différentes sources de chaleur. Ils débitent sur des résistances de 22 ou $33 \Omega$ sous 24 Volts, ce qui donne une puissance maximale de $88 \mathrm{~W}$, largement suffisante par rapport à la valeur calculée de $55 \mathrm{~W}$.

- 2 systèmes de ventilation. Le premier, dans le bâtiment, permet de brasser l'air pour assurer une bonne convection et éviter la stratification thermique de l'air. Le deuxième, à l'extérieur, permet d'augmenter les échanges thermiques entre 
le bâtiment et son environnement. Ces 2 ventilateurs tournent pour l'instant à vitesse fixe, cependant une nouvelle carte sera ajoutée pour obtenir des vitesses de rotation variables.

Ces 6 actionneurs peuvent être activés ou désactivés, contrôlés ou non en fonction des différentes questions du TP.

Les 2 sorties du CNA de la carte de commande ne permettent pas de commander l'ensemble de ces 6 actionneurs. Un système de multiplexage des commandes a été réalisé pour pallier ce problème (fig 4).

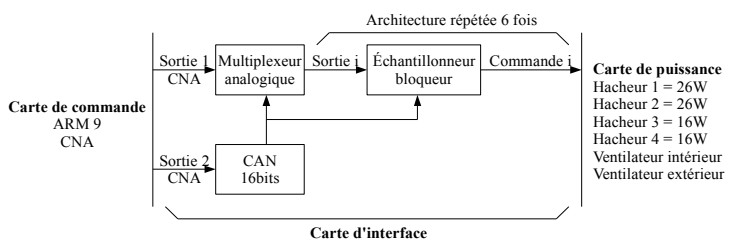

fig 4: Multiplexage des commandes.

\subsection{La carte d'acquisition}

$\mathrm{Ne}$ pas avoir pu intégrer de sources EnR (géothermique, solaire thermique ou éolienne), a sensiblement simplifié l'électronique d'acquisition. Celle-ci comporte un ensemble de capteurs de température (au nombre de 10) permettant de connaître la température extérieure et la température intérieure. La répartition réglable interne des capteurs en position va permettre aux étudiants d'optimiser le nombre de capteurs à utiliser ainsi que leurs positions pour assurer la meilleur régulation de température dans la maquette.

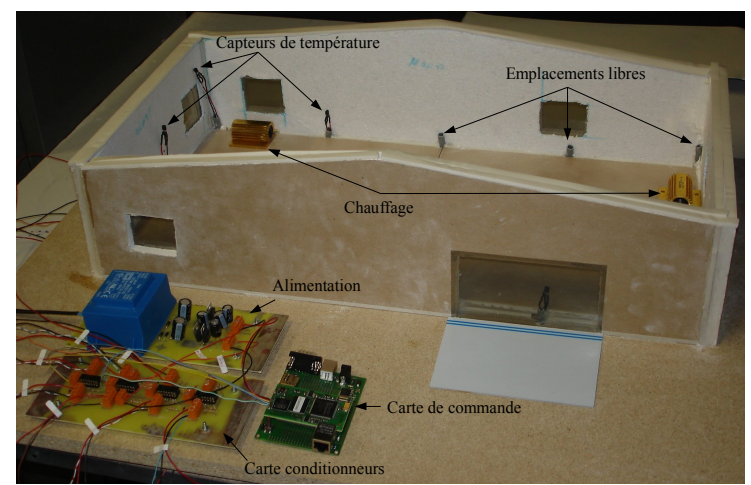

fig 5: Le système complet.

\section{TP AUTOMATIQUE LOGIQUE FLOUE}

Le système développé a permis de concevoir plusieurs travaux pratiques d'automatique. Les sujets proposés s'adressent aussi bien à des étudiants suivant le cursus de la licence SPTI/parcours EEA que des étudiants du master EAI et sont donc établis en fonction du niveau de chaque formation.

L'enseignement d'automatique en master s'oriente vers les techniques de l'Intelligence Artificielle, et plus particulièrement vers les réseaux de neurones et la logique floue, en commande et en supervision. Nous proposons donc d'illustrer le déroulement type d'un TP de « commande avancée » (master EAI $1^{\text {ère }}$ année) sur le contrôle de la température intérieure d'un habitat, par logique floue (il ne sera pas effectué de comparaison directe entre le comportement thermique de la maquette et un habitat réel). Après une étude théorique détaillée du système, les différentes étapes de la manipulation sont les suivantes :

\subsection{Réalisation du contrôleur flou}

Le contrôle flou est une extension du contrôle logique, qui s'appuie sur des règles linguistiques permettant une plus grande souplesse dans les définitions [7]. Ces règles sont du type :

SI température1 Grande Positive (entrée1)

ET/OU température2 Moyenne Positive (entrée2)

ALORS chauffage Grand (sortie)

On associe les termes linguistiques à des sous-ensembles flous qui peuvent être de formes trapézoïdaux, triangulaires ou gaussiens. Le raisonnement n'est pas logique au sens physique du terme. Les variables sont quantifiées par un degré d'appartenance compris entre 0 et 1 [8] pour chaque sous-ensemble flou.

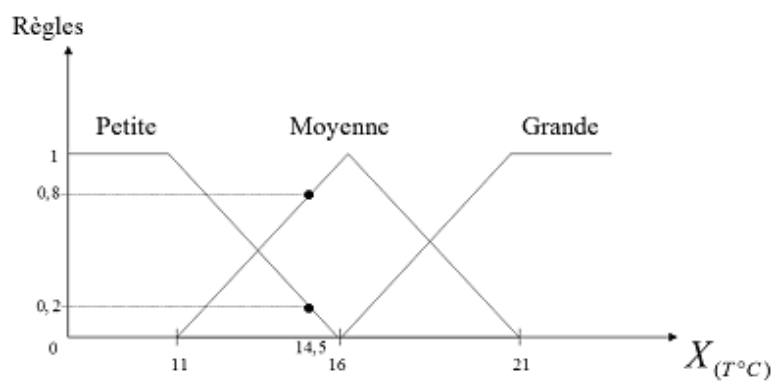

fig 6: Exemple de sous-ensembles flous triangulaires / trapézoïdaux.

Il s'agit donc, comme l'illustre l'exemple de la figure 6 de définir la variable $\mathrm{X}$ (température intérieure, par exemple $14,5^{\circ} \mathrm{C}$ ) dont l'univers de discours est $\left[0 ; 35^{\circ} \mathrm{C}\right]$, comme appartenant au sous-ensemble flou «Petite» avec un degré d'appartenance de 0,2 et au sous-ensemble flou «Moyenne» avec un degré d'appartenance de 0,8 .

Le contrôle flou n'est pas obligatoirement soumis à une seule entrée. En effet, il est possible de prendre en compte un nombre d'entrées important (par exemple la totalité des capteurs). La logique floue permet de mieux appréhender des grandeurs imprécises ou incertaines, $\mathrm{c}^{\prime}$ est un outil efficace lorsque les systèmes étudiés sont soumis à de fortes incertitudes. L'écriture des règles rend plus simple la prise en compte d'une expertise humaine sur le système à contrôler.

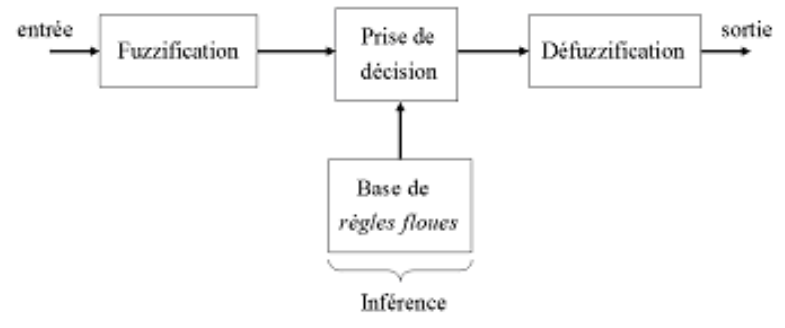

fig 7: Structure d'un contrôleur flou. 
La structure généralement employée pour un contrôleur flou est constituée de trois parties : la fuzzification, les règles d'inférence et la défuzzification (figure 7).

- La fuzzification : les variables d'entrée et de sortie choisies pour modéliser et commander un système sont des grandeurs numériques. L'étape de fuzzification consiste à transformer les grandeurs physiques en variables linguistiques (variables floues) en vue d'un traitement d'inférence. Une fois le choix des variables d'entrée et de sortie réalisé en fonction du problème à traiter, des ensembles flous définis sur leurs univers sont associés à chaque variable. A ces ensembles flous correspondent des labels linguistiques et des fonctions d'appartenance. Ces labels linguistiques seront utilisés pour écrire les règles d'inférence.

- Les inférences floues : la description de l'évolution d'un système (modélisation) et d'une commande (contrôle de procédé) se fait par l'intermédiaire de règles floues. Le but de l'inférence floue (ou évaluation des règles) est de déterminer les sorties du contrôleur à partir des entrées floues issues de la fuzzification des entrées réelles et des règles de commande. Les sorties intermédiaires issues de l'évaluation des règles sont encore floues.

- L'étape de défuzzification consiste à transformer l'information floue en une valeur numérique, afin de la transmettre au système. Il existe plusieurs approches pour réaliser cette opération, les plus employées sont les méthodes du centre de gravité et de la moyenne des maxima.

\subsection{Résultats et analyses}

Ce schéma illustre une structure simple avec une entrée, une sortie et une seule boucle de rétroaction. Mais on a souvent dans la pratique plusieurs variables d'entrée et une commande parallèle agissant sur plusieurs sorties.

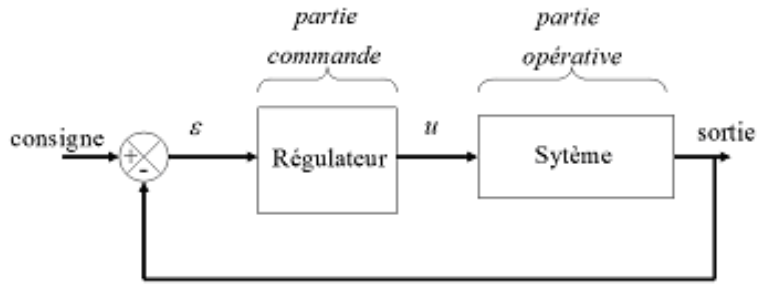

fig 8: Structure de la boucle de commande.

Le contrôleur flou infère les ordres de commande de la partie opérative (ou processus) à partir des règles de commande et des valeurs observées des termes linguistiques représentés par leurs fonctions d'appartenance. La consigne représente la température intérieure souhaitée. Il s'agit de définir des scénarii conventionnels en terme de consigne de température caractérisant les types de bâtiments. Les tableaux 1 et 2, respectivement scénarii d'occupation et température de consigne en chauffage sont deux des principaux critères de la caractérisation du climat et des scénarii « règles de calcul TH-CE $2005 »$ [2]. Il faut les combiner avec le type d'usage de bâtiment, par exemple, on définit pour un logement son «horaire d'occupation » par le label linguistique «Long", et son «niveau de température » par le label linguistique « Moyen ».

\begin{tabular}{|c|c|c|}
\hline $\begin{array}{c}\text { Horaires } \\
\text { d'occupa- } \\
\text { tion }\end{array}$ & Heures par jour & Jours par semaine \\
\hline Longs & $\begin{array}{c}16(\text { de } 0 \mathrm{~h} \text { à } 10 \mathrm{~h} \text { et } \\
\text { de } 18 \mathrm{~h} \text { à } 24 \mathrm{~h})\end{array}$ & 5 (lundi au vendredi) \\
\cline { 2 - 3 } & $24 \mathrm{~h}$ & Samedi et dimanche \\
\hline Moyens & $10(8 \mathrm{~h}$ à $18 \mathrm{~h})$ & 5 (lundi au vendredi) \\
\hline Courts & $5(9 \mathrm{~h}$ à $14 \mathrm{~h})$ & 5 (lundi au vendredi) \\
\hline
\end{tabular}

Tableau 1: Scénarii d'occupation

\begin{tabular}{|c|c|c|c|}
\hline $\begin{array}{c}\text { Niveau de } \\
\text { tempéra- } \\
\text { ture }\end{array}$ & $\begin{array}{c}\text { Occupa- } \\
\text { tion }\end{array}$ & $\begin{array}{c}\text { Réduction de } \\
\text { chauffage de } \\
\text { moins de } \mathbf{4 8 h}\end{array}$ & $\begin{array}{c}\text { Réduction de } \\
\text { chauffage } \\
\text { de plus de } \mathbf{4 8 h}\end{array}$ \\
\hline Élevé & $21^{\circ} \mathrm{C}$ & $18^{\circ} \mathrm{C}$ & $7^{\circ} \mathrm{C}$ \\
\hline Moyen & $19^{\circ} \mathrm{C}$ & $16^{\circ} \mathrm{C}$ & $7^{\circ} \mathrm{C}$ \\
\hline Réduit & $15^{\circ} \mathrm{C}$ & $7^{\circ} \mathrm{C}$ & $7^{\circ} \mathrm{C}$ \\
\hline
\end{tabular}

Tableau 2: Températures de consigne conventionnelles en chauffage

Il est donc possible de concevoir un scénario sur le principe suivant :

- Niveau de température Moyen $\left(19^{\circ} \mathrm{C}\right)$ pour horaires d'occupation Moyens (16h d'occupation du lundi au vendredi)

- Niveau de température Élevé $\left(21^{\circ} \mathrm{C}\right)$ pour horaires d'occupation Longs (du samedi au dimanche)

L'étape finale du TP est d'ajuster les paramètres du contrôleur, par exemple les règles floues ou les méthodes d'inférence, pour obtenir un système optimal capable de suivre le cahier des charges. L'étudiant doit alors vérifier, par des méthodes classiques (Bode, Nyquist, etc.), la dynamique, la précision et la robustesse du contrôleur développé.

\section{CONCLUSIONS ET PERSPECTIVES}

Cet article a présenté une version simplifiée d'un sujet de TP relatif au contrôle par logique floue. L'avantage d'un tel système est qu'il est possible de tester un grand nombre de technique de régulation (PID, TOR, RNA, etc...). En ce qui concerne la logique floue, les TP suivants permettront de développer d'autres contrôleurs comme par exemple :

- Un PID-flou : les paramètres du PID sont calculés par logique floue.

- Une commande prédictive : le contrôleur est combiné à un modèle prédictif flou du système permettant de compenser le retard pur du système.

L'ensemble de ces travaux pratiques permet d'aborder de façon précise les différents aspects des techniques de l'Intelligence Artificielle. Cette maquette a été conçue

1 Température de consigne utilisée lors d'une inoccupation des locaux de plus ou moins $48 \mathrm{~h}$. 
afin d'être également utilisée pour les travaux pratiques de capteurs et d'électronique embarquée, TP qui n'ont pas pu être développés ici. De plus, ce système est un outil pédagogique essentiel pour une formation comme la notre (Electronique embarquée, et gestion des EnR pour le bâtiment) car elle est conçue de manière à se rapprocher le plus possible d'un système réel et permet d'englober tous les aspects de l'EEA.

\section{BIBLIOGRAPHIE}

[1] Journal Officiel, (2006). Arrêté du 19 juillet 2006 relatif aux indices Th-CE 2005, République française.

[2] ADEME (Agence De l'Environnement et de la Maîtrise de l'Energie) (2007), "Les chiffres clés $d u$ bâtiment en 2006 », Publications de l'ADEME.

[3] Journal Officiel (2006). Décret $n^{\circ} 2006-592 d u$ 24 mai 2006 relatifs aux caractéristiques thermiques et à la performance énergétique des constructions, République française.

[4] Journal Officiel (2006), Décret $n^{\circ} 2006-1147 d u$ 14 septembre 2006 relatif au diagnostic de performance énergétique et à l'état de l'installation intérieure, République française.

[5] http://www.armadeus.com/

[6] Thierry Talbert, Matthieu Caussanel, Arnaud Perona, Monique Polit, Julien Boibessot, Nicolas Colombain, "L'électronique embarquée des systèmes EnR en enseignement ", CETSIS 2007, Bordeaux

[7] H. Hellendoorn and D, Driankov, 1997, « Fuzzy model identification, selected approaches ", SpringerVerlag Berlin Heidelberg, ISBN 3-540-62721-9, New York.

[8] Adama traore, Stéphane Grieu, Frédérik Thiéry, Monique Polit and Jesus Colprim, 2006, " Control of a sludge height in a secondary settler using fuzzy algorithm », Computer and Chemical engineering, 